\title{
Functoriality and number of solutions of congruences
}

\author{
by \\ Henry H. Kim (Toronto and Seoul)
}

In this note we use Langlands functoriality to prove certain results on the number of solutions of congruences, complementing results in [F1-3]. We would like to thank the referee for pointing out a mistake.

1. Number of solutions of congruences. Let $f(x)=x^{d}+a_{1} x^{d-1}+$ $\cdots+a_{d}, a_{1}, \ldots, a_{d} \in \mathbb{Z}$ be an irreducible polynomial. Let $N_{f}(n)$ be the number of solutions of $f(x) \equiv 0(\bmod n)$. It is an important problem to study $N_{f}(n)$. Let $L$ be the splitting field of $f$ with the Galois group $G$. Let $E=\mathbb{Q}[\alpha]$, where $\alpha$ is a root of $f$. Then $[E: \mathbb{Q}]=d$. Let $\operatorname{Gal}(L / E)=H$. Let $S(L / \mathbb{Q})=\left\{p: N_{f}(p)=d\right\}$. Then it is known that $S(L / \mathbb{Q})$ determines $L$ completely. It is a goal of the class field theory to determine the set $S(L / \mathbb{Q})$. Except for finitely many primes, $p \in S(L / \mathbb{Q})$ if and only if $p$ splits completely in $L$ : this comes from the fact that $p$ splits completely in $L$ if and only if $p$ splits completely in $E$. It is clear that if $p$ splits completely in $L$, then $p$ splits completely in $E$. Conversely, let $\mathfrak{P}, \mathfrak{p}$ be primes in $L, E$, respectively, such that $\mathfrak{P}|\mathfrak{p}, \mathfrak{p}| p$. Then $p$ splits completely in $L$ if and only if $L_{\mathfrak{P}}=\mathbb{Q}_{p}$. We fix an embedding $\mathbb{Q}_{p} \hookrightarrow \overline{\mathbb{Q}}_{p}$. Suppose $p$ splits completely in $E$. Then for any $\mathfrak{p} \mid p, E \subset E_{\mathfrak{p}}=\mathbb{Q}_{p} \hookrightarrow \overline{\mathbb{Q}}_{p}$. So every conjugate of $E$ is contained in $\mathbb{Q}_{p}$. Hence $L$ is contained in $\mathbb{Q}_{p}$. So $p$ splits completely in $L$. By the well-known theorem of Dedekind (e.g. [N, Theorem 4.33]), except for finitely many primes (in fact, if $p$ does not divide the discriminant of $f(x)$, or $\left.\left(\mathcal{O}_{E}: \mathbb{Z}[\alpha]\right)\right), p$ splits completely in $E$ if and only if $N_{f}(p)=d$.

Consider $\operatorname{Ind}_{H}^{G} 1=1+\varrho$, where $\varrho: G \rightarrow \mathrm{GL}_{d-1}(\mathbb{C})$ is a direct sum of non-trivial irreducible representations of $G$, i.e.,

$$
\varrho=n_{1} \varrho_{1}+\cdots+n_{k} \varrho_{k}
$$

where $\varrho_{1}, \ldots, \varrho_{k}$ are non-trivial irreducible representations of $G$. By Frobenius reciprocity, we see that if $\varrho_{i}$ is a 1-dimensional character, then $n_{i}=1$.

2000 Mathematics Subject Classification: 11N37, 11F70.

Partially supported by NSERC grant. 
(See $[\mathrm{FH}]$, for example.) We include the case $H=\{1\}$. In particular, if $G$ is abelian, then $\mathbb{Q}[\alpha] / \mathbb{Q}$ is Galois, and hence $E=L$, and $H=\{1\}$.

The Artin conjecture asserts that $\zeta_{E}(s) / \zeta(s)$ is entire. Langlands functoriality (the strong Artin conjecture) predicts that there exists an automorphic representation $\pi=\bigotimes \pi_{p}$ of $\mathrm{GL}_{d-1}(\mathbb{A})$ which corresponds to $\varrho$. If $\varrho$ is irreducible, then $\pi$ is cuspidal [R2]. More precisely, let $\pi_{i}$ be a cuspidal representation corresponding to $\varrho_{i}$. Then $\pi$ is the isobaric sum

$$
\pi=\underbrace{\pi_{1} \boxplus \cdots \boxplus \pi_{1}}_{n_{1}} \boxplus \cdots \boxplus \underbrace{\pi_{k} \boxplus \cdots \boxplus \pi_{k}}_{n_{k}} .
$$

In particular, the Langlands-Tunnell theorem says that if $\varrho$ is a 2-dimensional representation with solvable image, then the strong Artin conjecture is true.

If $p$ is unramified, then $\varrho\left(\operatorname{Frob}_{p}\right)$ is the semisimple conjugacy class of $\pi_{p}$. Let $\operatorname{diag}\left(\alpha_{1 p}, \ldots, \alpha_{d-1, p}\right)$ give rise to the semisimple conjugacy class of $\pi_{p}$, and let $a_{p}=\alpha_{1 p}+\cdots+\alpha_{d-1, p}$. In particular, we have the $L$-function (without the $\Gamma$-factors)

$$
L(s, \pi)=\prod_{p} L\left(s, \pi_{p}\right)=\sum_{n=1}^{\infty} \frac{a_{n}}{n^{s}}
$$

such that $\zeta_{E}(s)=\zeta(s) L(s, \pi)$.

We prove that if $\sigma=\operatorname{Ind}_{H}^{G} 1$, then $\chi_{\sigma}\left(\operatorname{Frob}_{p}\right)=N_{f}(p)$, so that $N_{f}(p)=$ $1+a_{p}$. We can see this in two ways. First, by the property of the Artin $L$-function, $L\left(s, \operatorname{Ind}_{H}^{G} 1, L / \mathbb{Q}\right)=\zeta_{E}(s)$. Let $\zeta_{E}(s)=\prod_{p} L_{p}(s)$. If $N_{f}(p)=a$, then $L_{p}(s)$ has the form $\left(1-p^{-s}\right)^{-a} \prod_{i=1}^{r}\left(1-p^{-k_{i} s}\right)^{-1}$, where $k_{i} \geq 2$. Hence $L_{p}(s)=\left(1-a p^{-s}+\cdots \pm p^{-d s}\right)^{-1}$.

Second, $\sigma=\operatorname{Ind}_{H}^{G} 1$ is the permutation representation of $G$ on the left cosets of $H$ in $G$. Let $\left\{g_{i} H: i=1, \ldots, d\right\}$ be the left cosets. Then $\chi_{\sigma}(g)$ is the trace of the permutation matrix given by $g_{i} H \mapsto g g_{i} H$. It is the number of the left cosets such that $g_{i}^{-1} g g_{i} \in H$. Suppose $p$ decomposes as $p \mathcal{O}_{E}=\mathfrak{p}_{1} \cdots \mathfrak{p}_{k}$ such that each $\mathfrak{p}_{i}$ is unramified, and has the residual degree $f_{i}$. Then $d=f_{1}+\cdots+f_{k}$. If $\mathfrak{P} \mid \mathfrak{p}_{i}$, then $\left(\frac{L / E}{\mathfrak{P}}\right)=\left(\frac{L / \mathbb{Q}}{\mathfrak{P}}\right)^{f_{i}} \in H$. Hence $\left(\frac{L / \mathbb{Q}}{\mathfrak{P}}\right) \in H$ if and only if $f_{i}=1$. Pick $\mathfrak{P}_{i} \mid \mathfrak{p}_{i}$ for each $i$. Pick elements $\tau_{i}$ which send $\mathfrak{p}_{1}$ to $\mathfrak{p}_{i}$ for $i=1, \ldots, k$. Then $\tau_{i}\left(\frac{L / \mathbb{Q}}{\mathfrak{P}_{i}}\right)^{k_{i}}, i=1, \ldots, k, 0 \leq k_{i} \leq f_{i}-1$, are coset representatives [N, Theorem 7.29]. Hence $\chi_{\sigma}\left(\right.$ Frob $\left._{p}\right)$ is the number of $i$ 's such that $f_{i}=1$. It is exactly $N_{f}(p)$. So $\chi_{\sigma}\left(\operatorname{Frob}_{p}\right)=N_{f}(p)$.

Since $\pi$ is an automorphic representation of $\mathrm{GL}_{d-1}(\mathbb{A}), L(s, \pi)$ has an analytic continuation to all of $\mathbb{C}$, and satisfies an appropriate functional equation. Hence we have 
Proposition 1 ([FI]). Let $a_{n}$ be as above. Then

$$
\sum_{n \leq x} a_{n}=O\left(x^{(d-2) / d+\varepsilon}\right) .
$$

Hence the series $\sum_{n=1}^{\infty} a_{n} / n^{s}$ converges for $\operatorname{Re}(s)>(d-2) / d+\varepsilon$. In particular,

$$
\sum_{p \leq x} \frac{a_{p}}{p}=O(1)
$$

2. Distribution of values of $r_{2}(n)$. Let $r_{2}(n)=\sum_{x_{1}^{2}+x_{2}^{2}=n} 1$. We are interested in

$$
\sum_{n \leq x} r_{2}(f(n))
$$

If $f(x)=a x^{2}+b x+c$, then (see [F2] for the details)

$$
\sum_{n \leq x} r_{2}(f(n))= \begin{cases}A(f) x \log x+O(x \log \log x) & \text { if } b^{2}-4 a c=-\mu^{2} \\ B(f) x+O\left(x^{8 / 9}(\log x)^{3}\right) & \text { if } b^{2}-4 a c \neq-\mu^{2}\end{cases}
$$

We use (see [F2] for the precise reference)

LEMma 2. Let $t(n)$ be a multiplicative function such that $t(n) \geq 0$ and $t\left(p^{k}\right) \ll k^{c}, k \in \mathbb{N}$ (p prime, and c constant $)$. Let $f(x)=\sum_{i=0}^{l} a_{i} x^{i} \in \mathbb{Z}[x]$ be irreducible such that $\left(a_{0}, \ldots, a_{l}\right)=1$. Then

$$
\sum_{n \leq x} t(f(n)) \ll x \exp \left(\sum_{p \leq x} \frac{N_{f}(p)(t(p)-1)}{p}\right),
$$

where the implied constant depends on $t(n)$ and $f(n)$.

Hence we need to compute

$$
\sum_{p \leq x} \frac{N_{f}(p)\left(r_{2}(p)-1\right)}{p}
$$

Here we have removed finitely many primes $p$ where $\pi_{p}$ is not spherical, or $p=2$. However, if $p \neq 2$, then $r_{2}(p)=1+\left(\frac{-1}{p}\right)$. Let $\chi_{4}$ be the nontrivial character of $(\mathbb{Z} / 4 \mathbb{Z})^{\times}$. Then $\chi_{4}(p)=\left(\frac{-1}{p}\right)$. Since $\sum_{n=1}^{\infty} \chi_{4}(n) / n^{s}$ is holomorphic at $s=1, \sum_{p \leq x} \chi_{4}(p) / p=O(1)$. Also

$$
\sum_{n=1}^{\infty} \frac{a_{n} \chi_{4}(n)}{n^{s}}=L\left(s, \pi \otimes \chi_{4}\right) \text {. }
$$

If $\pi$ is not cuspidal, and $\chi_{4}$ occurs in the decomposition (1), then it occurs with multiplicity one, and hence $L\left(s, \pi \otimes \chi_{4}\right)$ has a simple pole at $s=1$. So $\sum_{p \leq x} a_{p} \chi_{4}(p) / p=\log \log x+O(1)$. Otherwise, $L\left(s, \pi \otimes \chi_{4}\right)$ is holomorphic at $s=1$, and $\sum_{p \leq x} a_{p} \chi_{4}(p) / p=O(1)$. Here we note that $\chi_{4}$ occurs in 
the decomposition (1) if and only if $\chi_{4}$ is an irreducible character of $G$ and $\left.\chi_{4}\right|_{H}=1$. Hence it is the case if and only if $\mathbb{Q}[\sqrt{-1}] \subset E$. Hence

$$
\sum_{p \leq x} \frac{N_{f}(p)\left(r_{2}(p)-1\right)}{p}= \begin{cases}\log \log x+O(1) & \text { if } \mathbb{Q}[\sqrt{-1}] \subset E \\ O(1) & \text { otherwise }\end{cases}
$$

Therefore, we obtain

Theorem 3. Suppose we have the strong Artin conjecture for $L(s, \varrho)$. Then

$$
\sum_{n \leq x} r_{2}(f(n)) \ll \begin{cases}x \log x & \text { if } \mathbb{Q}[\sqrt{-1}] \subset E, \\ x & \text { otherwise. }\end{cases}
$$

If $f(x)=a x^{2}+b x+c, b^{2}-4 a c=-\mu^{2}$, then $E=\mathbb{Q}[\sqrt{-1}]$, and hence $\zeta_{E}(s)=\zeta(s) L\left(s, \chi_{4}\right)$. So the estimate $(2)$ is the best possible. If $f(x)$ is the $m$ th cyclotomic polynomial, and $4 \mid m$, then $\mathbb{Q}[\sqrt{-1}] \subset E=\mathbb{Q}\left[e^{2 \pi i / m}\right]$. Hence $\sum_{n \leq x} r_{2}(f(n)) \ll x \log x$.

We give five examples which satisfy the condition in Theorem 3 .

Example 1. Suppose $f(x)=x^{3}+a x^{2}+b x+c$, and its Galois group is $S_{3}$ with the discriminant $D$. Then $\varrho: S_{3} \rightarrow \mathrm{GL}_{2}(\mathbb{C})$ is the irreducible 2dimensional representation. Hence $\varrho$ gives rise to a cuspidal representation $\pi$ of $\mathrm{GL}_{2}\left(\mathbb{A}_{\mathbb{Q}}\right)$. Let $L(s, \pi)=\sum_{n=1}^{\infty} a_{n} n^{-s}$. Then $N_{f}(p)=1+a_{p}$. In particular, if $\varrho$ is odd, i.e., $D<0$, it comes from a holomorphic cusp form $F$ of weight 1 and level $|D|$. Then $F(z)=\sum_{n=1}^{\infty} a_{n} q^{n}, q=e^{2 \pi i z}$. In this case, $S(L / \mathbb{Q})=$ $\left\{p: a_{p}=2,\left(\frac{D}{p}\right)=1\right\}$ and $\sum_{n \leq x} r_{2}(f(n)) \ll x$.

EXAMPLE 2. Let $f(x)=x^{4}+a x^{3}+b x^{2}+c x+d$, and assume its Galois group is $S_{4}$ with discriminant $D$. Here $\varrho: S_{4} \rightarrow \mathrm{GL}_{3}(\mathbb{C})$ is one of the two irreducible 3-dimensional representations. There exists a Galois extension $\widetilde{L} / \mathbb{Q}$ such that $\operatorname{Gal}(\widetilde{L} / \mathbb{Q}) \simeq \mathrm{GL}_{2}\left(\mathbb{F}_{3}\right)$, and $[\widetilde{L}: L]=2$. Then $\varrho=\operatorname{Sym}^{2}(\sigma)$, where $\sigma$ is the 2-dimensional representation $\sigma: \mathrm{GL}_{2}\left(\mathbb{F}_{3}\right) \rightarrow \mathrm{GL}_{2}(\mathbb{C})$ (see [Ki2] for the details). Since $\mathrm{GL}_{2}\left(\mathbb{F}_{3}\right)$ is solvable, by the Langlands-Tunnell theorem, $\sigma$ gives rise to a cuspidal representation $\pi$ (if $D<0$, it is odd and it comes from a holomorphic cusp form of weight 1$)$. Let $L(s, \pi)=$ $\sum_{n=1}^{\infty} b_{n} n^{-s}$. Then the central character is $\omega_{\pi}(p)=\left(\frac{p}{D}\right)$. Then $\varrho$ gives rise to the Gelbart-Jacquet lift $\operatorname{Sym}^{2}(\pi)$ and $a_{p}=b_{p}^{2}-\omega_{\pi}(p)$. Hence $N_{f}(p)=$ $1+b_{p}^{2}-\left(\frac{p}{D}\right)$. Since $\sigma$ is not of dihedral type, $\operatorname{Sym}^{2}(\pi)$ is cuspidal.

In this case, $S(L / \mathbb{Q})=\left\{p: a_{p}= \pm 2,\left(\frac{p}{D}\right)=1\right\}$, and $\sum_{n \leq x} r_{2}(f(n)) \ll x$.

EXAMPLE 3. Let $f(x)=x^{4}+a x^{3}+b x^{2}+c x+d$ with Galois group $A_{4}$ and discriminant $D$. Here $\varrho: S_{4} \rightarrow \mathrm{GL}_{3}(\mathbb{C})$ is the irreducible 3-dimensional representation. In this case, there exists a Galois extension $\widetilde{L} / \mathbb{Q}$ such that $\operatorname{Gal}(\widetilde{L} / \mathbb{Q}) \simeq \operatorname{SL}_{2}\left(\mathbb{F}_{3}\right)$, and $[\widetilde{L}: L]=2$. Then $\varrho=\operatorname{Sym}^{2}(\sigma)$, where $\sigma$ is the 
2-dimensional representation $\sigma: \mathrm{SL}_{2}\left(\mathbb{F}_{3}\right) \rightarrow \mathrm{GL}_{2}(\mathbb{C})$. This is similar to the $S_{4}$ case.

EXAMPLE 4. Let $f(x)=x^{5}+a x^{4}+b x^{3}+c x^{2}+d x+e$ with Galois group $A_{5}$ and discriminant $D$. Here $\varrho: A_{5} \rightarrow \mathrm{GL}_{4}(\mathbb{C})$ is the irreducible 4dimensional representation. There exists a Galois extension $\widetilde{L} / \mathbb{Q}$ such that $\operatorname{Gal}(\widetilde{L} / \mathbb{Q}) \simeq \mathrm{SL}_{2}\left(\mathbb{F}_{5}\right)$, and $[\widetilde{L}: L]=2$. Then $\varrho=\sigma \otimes \sigma^{\tau}$, where $\sigma$ is one of the 2-dimensional representations $\sigma: \mathrm{SL}_{2}\left(\mathbb{F}_{5}\right) \rightarrow \mathrm{GL}_{2}(\mathbb{C})$, and $\tau$ is the automorphism $\sqrt{5} \mapsto-\sqrt{5}$ (see [Ki1] for the details). Suppose $\sigma$ is odd, and it gives rise to a cuspidal representation $\pi$ which is attached to a holomorphic cusp form of weight $1, F(z)=\sum_{n=1}^{\infty} b_{n} q^{n}$. Then $\varrho$ gives rise to the functorial product $\pi \otimes \pi^{\tau}$ (see [R1]), and $a_{p}=b_{p} b_{p}^{\tau}$. Hence $N_{f}(p)=1+b_{p} b_{p}^{\tau}$.

R. Taylor $[\mathrm{T}]$ proved infinitely many cases of modularity of odd icosahedral Galois representations. In particular, the following quintic polynomials give rise to holomorphic cusp forms of weight 1 :

$$
\begin{aligned}
& x^{5}+2 x^{4}+6 x^{3}+8 x^{2}+10 x+8, \\
& x^{5}+6 x^{4}+x^{3}+4 x^{2}-24 x+32, \\
& x^{5}-2 x^{3}+2 x^{2}+5 x+6, \\
& x^{5}+5 x^{4}+8 x^{3}-20 x^{2}-21 x-5 .
\end{aligned}
$$

In this case, $S(L / \mathbb{Q})=\left\{p: b_{p}= \pm 2\right\}$, and $\sum_{n \leq x} r_{2}(f(n)) \ll x$.

EXAmple 5 ([F2]). Let $f(x)=x^{4}-m$, where $m$ is a positive integer which is not a square. Then $L=\mathbb{Q}\left[\sqrt{-1}, m^{1 / 4}\right]$. Let $E=\mathbb{Q}\left[m^{1 / 4}\right]$. Then we can show that $\operatorname{Ind}_{H}^{G} 1$ is the direct sum of the trivial character, one nontrivial 1-dimensional character $\chi$ and the unique 2-dimensional irreducible representation $\varrho$. Then $\varrho$ gives rise to a holomorphic cusp form $F$ of weight 1 . Let $F(z)=\sum_{n=1}^{\infty} a_{n} q^{n}, q=e^{2 \pi i z}$, and assume $\chi$ gives rise to a Dirichlet character $\chi(p)=\left(\frac{m_{0}}{p}\right)$, where $m_{0}$ is the square-free part of $m$. Then $N_{f}(p)=$ $1+\left(\frac{m_{0}}{p}\right)+a_{p}$. In this case, $S(L / \mathbb{Q})=\left\{p: a_{p}=2,\left(\frac{m_{0}}{p}\right)=1\right\}$, and $\sum_{n \leq x} r_{2}(f(n)) \ll x$.

3. The sum $\sum_{n \leq x}|b(f(n))|^{2}$. Let $\pi^{\prime}=\bigotimes_{p} \pi_{p}^{\prime}$ be a cuspidal representation of $\mathrm{GL}_{2}\left(\mathbb{A}_{\mathbb{Q}}\right)$, and let $L\left(s, \pi^{\prime}\right)=\prod_{p} L\left(s, \pi_{p}^{\prime}\right)=\sum_{n=1}^{\infty} b(n) n^{-s}$ (without the $\Gamma$-factor). Let $f$ be as in Section 1 . We assume that $L\left(s, \pi \times \operatorname{Ad}\left(\pi^{\prime}\right)\right)$ is holomorphic at $s=1$, where $\pi$ corresponds to $\varrho$. In particular, it is the case if $\pi^{\prime}$ is attached to a holomorphic cusp form of weight $k \geq 2$. Note that $L\left(s, \pi \times \operatorname{Ad}\left(\pi^{\prime}\right)\right)$ has a pole at $s=1$ if and only if $\pi \simeq \operatorname{Ad}\left(\pi^{\prime}\right)$. So it is very rare. 
We are interested in the sum

$$
\sum_{n \leq x}|b(f(n))|^{2}
$$

If the Ramanujan conjecture holds, then $|b(n)| \leq d(n)$, where $d(n)$ is the number of divisors of $n$, and we know that

$$
\sum_{n \leq x} d(f(n))^{2} \ll x(\log x)^{3} .
$$

(We can obtain this from Lemma 2 by observing that $\zeta(s)^{2}=\sum_{n=1}^{\infty} d(n) / n^{s}$. Namely, $d(n)$ is the Fourier coefficients for the automorphic representation $\pi^{\prime}=1 \boxplus 1 ; L\left(s, \pi^{\prime}\right)=\zeta(s)^{2}$. Then $L\left(s, \pi^{\prime} \times \pi^{\prime}\right)$ has a pole of order 4 at $s=1$. Hence $\sum_{p \leq x} d(p)^{2} / p=4 \log \log x+O(1)$ and $\sum_{p \leq x} a_{p} d(p)^{2} / p=O(1)$.)

This gives a trivial estimate (see [F3] for the details)

$$
\sum_{n \leq x}|b(f(n))|^{2} \ll x(\log x)^{3} .
$$

We would like to obtain a better estimate. Furthermore, we do not assume the Ramanujan conjecture for $\pi^{\prime}$.

THEOREM 4. Let $f, \varrho$ be as in Section 1. Suppose we have the strong Artin conjecture for $L(s, \varrho)$, and that $L\left(s, \pi \times \operatorname{Ad}\left(\pi^{\prime}\right)\right)$ is holomorphic at $s=1$, where $\pi$ corresponds to $\varrho$. Then

$$
\sum_{n \leq x}|b(f(n))|^{2} \ll x
$$

Proof. We follow [F3]. By Lemma 2, we need to compute

$$
\sum_{p \leq x} \frac{N_{f}(p)\left(|b(p)|^{2}-1\right)}{p} .
$$

Here we have removed finitely many primes $p$ where $\pi_{p}$ or $\pi_{p}^{\prime}$ is not spherical. Since $N_{f}(p)=1+a_{p}$, we need to consider $\sum_{p \leq x}|b(p)|^{2} / p$ and $\sum_{p \leq x} a_{p}|b(p)|^{2} / p$.

Since $L\left(s, \pi^{\prime} \times \tilde{\pi}^{\prime}\right)$ has a simple pole at $s=1$, we have $\sum_{p \leq x}|b(p)|^{2} / p=$ $\log \log x+O(1)$. Since the triple product $L$-function $L\left(s, \pi \times \pi^{\prime} \times \tilde{\pi}^{\prime}\right)=$ $L\left(s, \pi \times \operatorname{Ad}\left(\pi^{\prime}\right)\right) L(s, \pi)$ is holomorphic at $s=1$, we have $\sum_{p \leq x} a_{p}|b(p)|^{2} / p=$ $O(1)$. Since $\sum_{p \leq x} 1 / p=\log \log x+O(1)$ we get

$$
\sum_{p \leq x} \frac{N_{f}(p)\left(|b(p)|^{2}-1\right)}{p}=O(1) .
$$

We have the result unconditionally for the polynomials in the examples in Section 2. 
4. Distribution of values of $N_{f}(n)$. Let $f, \varrho$ be as in Section 1. In this section we do not need to assume the strong Artin conjecture for $L(s, \varrho)$. We are interested in the quantities

$$
\sum_{n \leq x} N_{f}(n), \quad \sum_{p \leq x} N_{f}(p) .
$$

Erdős proved (see $[\mathrm{F} 1]$ for the precise reference)

$$
\sum_{p \leq x} N_{f}(p)=\frac{x}{\log x}+O\left(\frac{x}{(\log x)^{2}}\right), \quad \sum_{p \leq x} \frac{N_{f}(p)}{p}=\log \log x+c(f)+o(1) .
$$

One can also show (see [F1] for the details)

$$
\sum_{n \leq x} N_{f}(n)=C(f) x+O\left(\frac{x}{(\log x)^{1 / 2-\varepsilon}}\right),
$$

where $C(f)=e^{-\gamma+c(f)} P$. Here $\gamma$ is the Euler constant and

$$
P=\prod_{p} e^{-N_{f}(p) / p}\left(1+\frac{N_{f}(p)}{p}+\frac{N_{f}\left(p^{2}\right)}{p^{2}}+\cdots\right) .
$$

We would like to obtain a better error term, following [F1]. Consider

$$
L(s)=\sum_{n=1}^{\infty} \frac{N_{f}(n)}{n^{s}} .
$$

Since $N_{f}(n)$ is multiplicative, we can write

$$
L(s)=\prod_{p}\left(1+\frac{N_{f}(p)}{p^{s}}+\frac{N_{f}\left(p^{2}\right)}{p^{2 s}}+\cdots\right)
$$

for $\operatorname{Re}(s)>1$. Here

$$
\zeta_{E}(s)=\prod_{p}\left(1-\frac{N_{f}(p)}{p^{s}}+\cdots \pm \frac{1}{p^{d s}}\right)^{-1} .
$$

Hence $L(s) / \zeta_{E}(s)$ is absolutely convergent for $\operatorname{Re}(s)>1 / 2$. Hence it is holomorphic and non-vanishing for $\operatorname{Re}(s)>1 / 2$. Let $L(s)=\zeta_{E}(s) A(s)$ for $\operatorname{Re}(s)>1$, where $A(s)$ is holomorphic and non-vanishing for $\operatorname{Re}(s)>1 / 2$. This provides the meromorphic continuation of $L(s)$ to $\operatorname{Re}(s)>1 / 2$. Since $\zeta_{E}(s)$ has a simple pole at $s=1, L(s)$ has a simple pole at $s=1$. We use Perron's formula:

$$
\sum_{n \leq x} N_{f}(n)=\frac{1}{2 \pi i} \int_{\alpha-i T}^{\alpha+i T} \frac{x^{s}}{s} L(s) d s+O\left(\frac{x^{1+2 \varepsilon}}{T}\right),
$$


for any $1 \leq T \leq x$, where $\alpha=1+\varepsilon$. We move the integration to the parallel segment with $\operatorname{Re}(s)=1 / 2+\varepsilon$. Then

$$
\sum_{n \leq x} N_{f}(n)=x \operatorname{Res}_{s=1} L(s)+\frac{1}{2 \pi i} \int_{1 / 2+\varepsilon-i T}^{1 / 2+\varepsilon+i T} \frac{x^{s}}{s} L(s) d s+O\left(\frac{x^{1+2 \varepsilon}}{T}\right) .
$$

We have the convexity bound for $\zeta_{E}(s)$ at $\operatorname{Re}(s)=1 / 2+\varepsilon$ (see $\left.[\mathrm{CN}]\right)$ :

$$
\left|\zeta_{E}(1 / 2+\varepsilon+i t)\right| \ll(1+|t|)^{d / 4} .
$$

Hence

$$
\frac{1}{2 \pi i} \int_{\alpha-i T}^{\alpha+i T} \frac{x^{s}}{s} L(s) d s \ll x^{1 / 2+\varepsilon} \int_{0}^{T} t^{d / 4-1} d t=O\left(x^{1 / 2+\varepsilon} T^{d / 4}\right) .
$$

Take $T=x^{2 /(d+4)}$. Then

$$
\sum_{n \leq x} N_{f}(n)=x \operatorname{Res}_{s=1} L(s)+O\left(x^{(d+2) /(d+4)+\varepsilon}\right) .
$$

We have proved

TheOREm 5. Let $f, L(s)$ be as above. Then $L(s)$ has a simple pole at $s=1$, and

$$
\sum_{n \leq x} N_{f}(n)=x \operatorname{Res}_{s=1} L(s)+O\left(x^{(d+2) /(d+4)+\varepsilon}\right) .
$$

Remark. Note that the above error estimate holds even when $G$ is abelian, or $G=S_{3}$, improving the result in [F1]. We have the convexity bounds for Dirichlet $L$-functions, namely, $|L(1 / 2+\varepsilon+i t, \chi)| \ll(1+|t|)^{1 / 6+\varepsilon}$. So if $G$ is abelian, $\left|\zeta_{E}(1 / 2+\varepsilon+i t)\right| \ll(1+|t|)^{d / 6+\varepsilon}$. Then the error bound is improved to $O\left(x^{(d+3) /(d+6)+\varepsilon}\right)$.

\section{References}

[CN] K. Chandrasekharan and R. Narasimhan, The approximate functional equation for a class of zeta-functions, Math. Ann. 152 (1963), 30-64.

[F1] O. M. Fomenko, The mean number of solutions of certain congruences, Zap. Nauchn. Sem. S.-Peterburg. Otdel. Mat. Inst. Steklov. (POMI) 254 (1998), 192-206 (in Russian); English transl.: J. Math. Sci. (New York) 105 (2001), 2257-2268.

[F2] - Distribution of values of Fourier coefficients for modular forms of weight 1, ibid. 226 (1996), 196-227 (in Russian); English transl.: ibid. 89 (1998), 1050-1071.

[F3] - Fourier coefficients of cusp forms and automorphic L-functions, ibid. 237 (1997), 194-226 (in Russian); English transl.: ibid. 95 (1999), 2295-2316.

[FI] J. Friedlander and H. Iwaniec, Summation formulae for coefficients of L-functions, Canad. J. Math. 57 (2005), 494-505.

[FH] W. Fulton and J. Harris, Representation Theory, Springer, 1991. 
[Ki1] H. Kim, An example of non-normal quintic automorphic induction and modularity of symmetric powers of cusp forms of icosahedral type, Invent. Math. 156 (2004), 495-502.

[Ki2] - On symmetric powers of cusp forms on $\mathrm{GL}_{2}$, in: The Conference on $L$-Functions, World Sci., 2007, 95-113.

[N] W. Narkiewicz, Elementary and Analytic Theory of Algebraic Numbers, Springer, 2004.

[R1] D. Ramakrishnan, Modularity of the Rankin-Selberg L-series, and multiplicity one for SL(2), Ann. of Math. 152 (2000), 45-111.

[R2] - Irreducibility and cuspidality, in: Representation Theory and Automorphic Forms, Progr. Math., Springer-Birkhäuser, to appear.

[T] R. Taylor, On icosahedral Artin representations II, Amer. J. Math. 125 (2003), 549-566.

Department of Mathematics

Korea Institute for Advanced Study

University of Toronto

Seoul, Korea

Toronto, ON M5S 2E4, Canada

E-mail: henrykim@math.toronto.edu

Received on 6.11.2006

and in revised form on 26.2.2007 